\title{
Colonic Perineurioma's Malignant Proximity to Serrated Colonic Polyps
}

\author{
Sindhura Kolli ${ }^{1}$, Srilaxmi Gujjula ${ }^{1}$, Mel A. Ona ${ }^{2}$ \\ 1. Internal Medicine, The Brooklyn Hospital Center, Affiliate of the Mount Sinai Hospital, Brooklyn, USA \\ 2. Gastroenterology, Pali Momi Medical Center, Honolulu, USA
}

$\square$ Corresponding author: Sindhura Kolli, sindhura.kolli@gmail.com

Disclosures can be found in Additional Information at the end of the article

\section{Abstract}

A colonic perineurioma is often considered a benign cousin to a colonic polyp. However, in the submucosal type of perineurioma, it is important to rule out the malignant gastrointestinal stromal tumor (GIST). Alternatively, in the BRAF-positive serrated types of perineuriomas, surveillance is equivalent to intervals designated to serrated polyps due to a similar malignant potential. These versions serve as reminders that colonic perineuriomas are not to be disregarded.

Categories: Internal Medicine, Gastroenterology, Oncology

Keywords: perineurioma, serrated polyp, colonic polyp, colon polyp, braf, gist

\section{Introduction}

This case examines the epidemiology, classification, clinical features, diagnosis, treatment, and most importantly, the differential diagnoses of a colonic perineurioma. Heeding the malignant potential of its variants and possible differentials influences the course of treatment, recurrence, and surveillance which is vital for a gastroenterologist.

\section{Case Presentation}

A 35-year-old Caucasian female presented with sharp, non-radiating, epigastric abdominal pain associated with alternating constipation and diarrhea. A computed tomography (CT) scan demonstrated a thickened stomach wall and diverticulitis at the splenic flexure. Vital signs and labs were within normal limits. Colonoscopy showed a unusual looking polyp in the transverse colon (Figure 1). 


\section{Cureus}

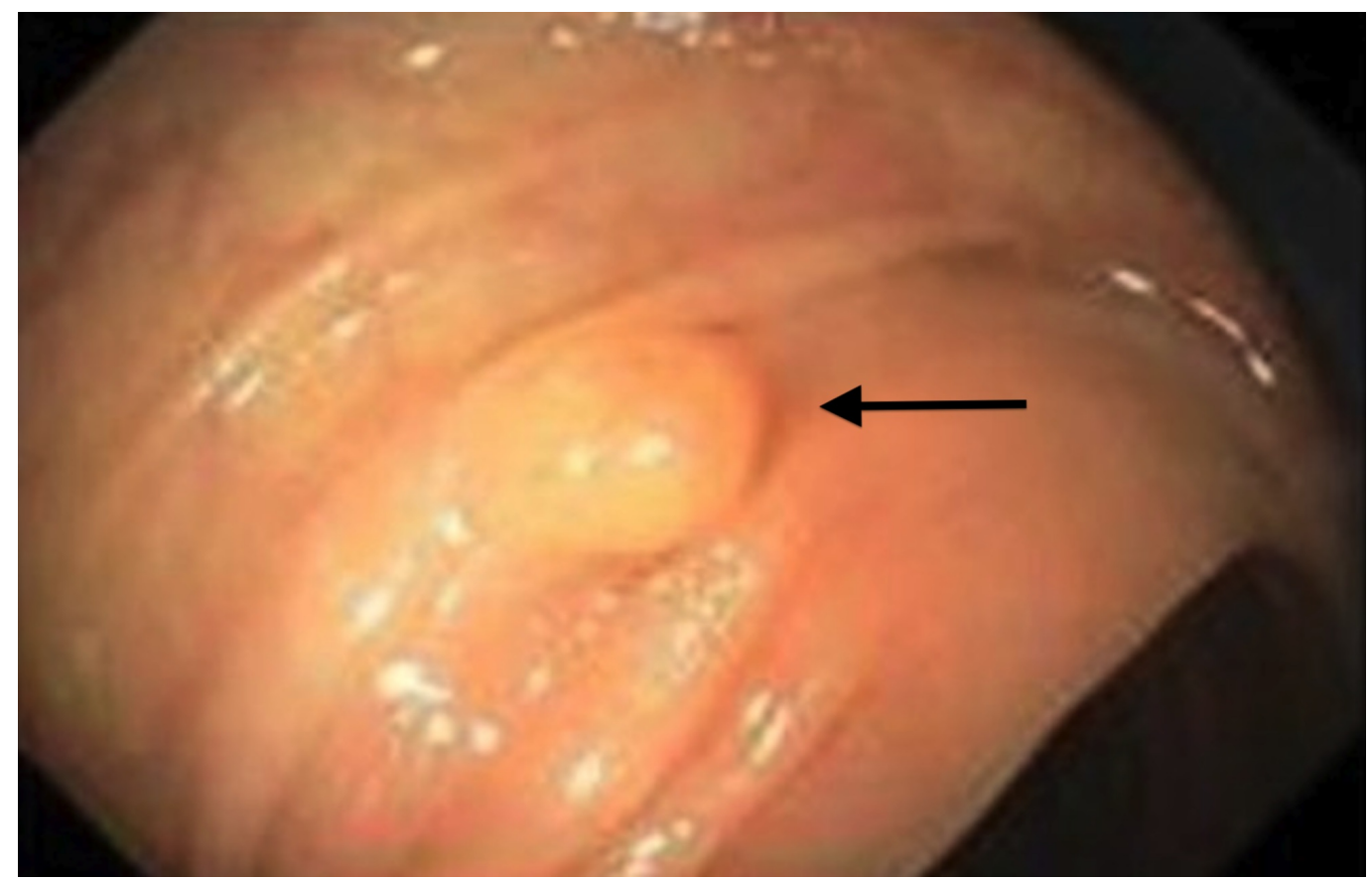

FIGURE 1: Polyp in the transverse colon

Narrow band imaging (NBI) did not demonstrate typical vessel appearance or polyp pit pattern (Figure 2). No other abnormalities were observed. Biopsies revealed a perineurioma. The patient was treated conservatively, her pain resolved, and she was discharged with plan for follow-up at recommended surveillance.

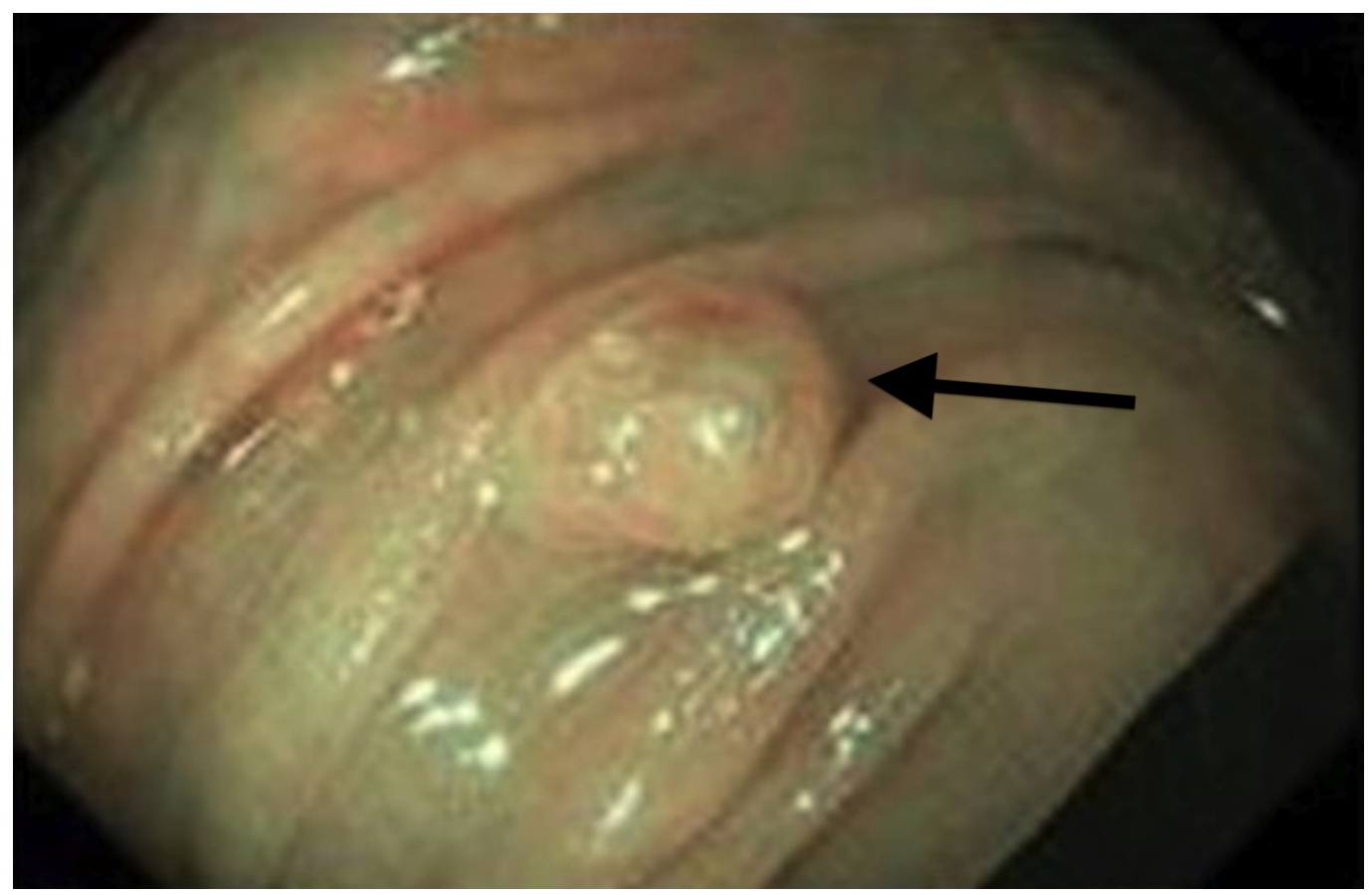

FIGURE 2: NBI showing unusual vessel appearance and surface pit pattern

$\mathrm{NBI}$, narrow band imaging 


\section{Discussion}

Colonic perineuriomas are rare benign mucosal lesions with a serrated or hyperplastic architecture composed of perineural cells. They are usually discovered incidentally during screening colonoscopies. With an incidence of $0.1 \%$ to $0.46 \%$, they are found distal to the splenic flexure in the sigmoid colon and the rectum mostly as polyps and rarely as solitary masses. No clear pathogenesis has been found. These polyps are considered benign reactive processes; however, the serrated variant confers malignant potential from BRAF mutations resulting in fibroblast differentiation and proliferation [1].

Mucosal perineuriomas are asymptomatic in comparison to the submucosal or intramural masses. Morphology alone can lead to strong suspicion of perineurioma, but the expression of at least two perineural markers, such as glucose transporter-1 (GLUT-1), Claudin-1, and epithelial membrane antigen (EMA), are generally required to confirm diagnosis [2]. Even though EMA stains are dependent on the antibody dilution and can be weak, Claudin-1 and GLUT-1 are considered to have high sensitivity and specificity to mark perineurial differentiation [3]. The mean age at diagnosis was 60 -years-old with a slight female majority [2].

Differential diagnosis is dependent on the origin of the lesion: intramucosal or submucosal. Neurofibroma, ganglioneuroma, schwannoma, neuroma, Schwann cell hamartoma, benign epithelioid nerve sheath tumor, and leiomyoma of the muscularis mucosae should be considered for intramucosal colorectal perineuriomas. For submucosal perineuriomas, schwannomas and the only malignant differential, a gastrointestinal stromal tumor (GIST) must be excluded [3]. Colorectal perineuriomas are considered to be benign. However, due to the malignancy potential in BRAF-positive serrated variants of colorectal perineurioma, we suggest abbreviated postpolypectomy surveillance repeat screening at similar intervals as serrated colorectal polyps [1].

\section{Conclusions}

A colonic perineurioma is considered a benign process. However, it is vital for gastroenterologists and patients to be aware of the malignant potential in BRAF-positive serrated variants and to rule out malignant differentials, such as GISTs, in submucosal perineuriomas.

\section{Additional Information \\ Disclosures}

Human subjects: Consent was obtained by all participants in this study. Brooklyn Hospital Center IRB Board issued approval $\mathrm{n} / \mathrm{a}$. Thank you for submitting the Publication materials for this project. The Brooklyn Hospital Center IRB has APPROVED your submission. No further action on this submission is required at this time. The following items are acknowledged in this submission: • Abstract/Summary-IRB-ColonPerineurioma • ApplicationForm-

CaseReportApplication - ConsentForm-CaseReportConsent If you have any questions, please contact Twyla Tate at 718-250-6947. Please include your project title in all correspondence with this committee. . Conflicts of interest: In compliance with the ICMJE uniform disclosure form, all authors declare the following: Payment/services info: All authors have declared that no financial support was received from any organization for the submitted work. Financial relationships: All authors have declared that they have no financial relationships at present or within the previous three years with any organizations that might have an interest in the submitted work. Other relationships: All authors have declared that there are no other 


\section{Cureus}

relationships or activities that could appear to have influenced the submitted work.

\section{References}

1. Jama GM, Evans M, Fazal MW, Singh-Ranger D: Perineurioma of the sigmoid colon . BMJ Case Rep. 2018, 2018:bcr-2018-227170. 10.1136/bcr-2018-227170

2. Van Wyk AC, Van Zyl H, Rigby J: Colonic perineurioma (benign fibroblastic polyp): case report and review of the literature. Diagn Pathol. 2018, 13:16. 10.1186/s13000-018-0694-Z

3. McCarthy AJ, Karamchandani DM, Chetty R: Neural and neurogenic tumours of the gastroenteropancreaticobiliary tract. J Clin Pathol. 2018, 71:565-578. 10.1136/jclinpath-2017204895 